TRABAJOS ORIGINALES

Rev Obstet Ginecol Venez. 2021; 81 (1): 39-48

https://doi.org/10.51288/00810108

\title{
Eficacia diagnóstica de la ecografía y la histeroscopia en pacientes con patología endometrial*
}

\author{
Drs. María Blanco, ${ }^{1}$ María Pacheco, ${ }^{2}$ Rafael Colmenares, ${ }^{3}$ Laura Vásquez. ${ }^{4}$
}

\begin{abstract}
RESUMEN
Objetivo: Determinar la eficacia diagnóstica de la ecografía y la histeroscopia en pacientes con patología endometrial que acudieron a la consulta de Ginecología del Hospital Universitario "Dr. Pedro Emilio Carrillo" enero 2017- julio 2017.

Método: Se realizó un estudio correlacional, cuasi-experimental y prospectivo, que incluyó 33 pacientes con patología endometrial a quienes se les realizó ecografia transvaginal, histeroscopia y toma de muestra para estudio histológico.

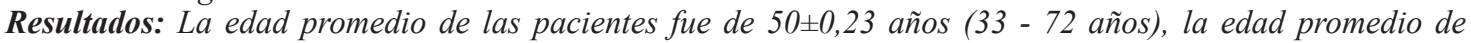

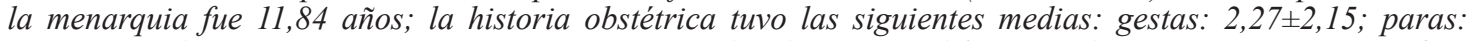

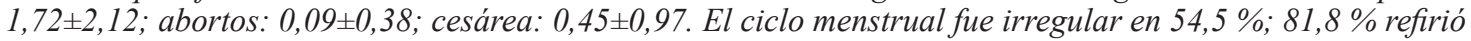
dismenorrea y 63,6\% utilizaron anticonceptivos orales. La mayoría cursó con dolor pélvico y antecedentes familiares como factor de riesgo. El diagnóstico ecográfico más frecuente fue engrosamiento endometrial. El diagnóstico histeroscópico más frecuente fue mioma submucoso (36,4\%). La ecografía transvaginal para el diagnóstico de miomas submucosos tiene $100 \%$ de especificidad y sensibilidad; la histeroscopia tiene 96 $\%$ de especificidad para pólipo endometrial, las lesiones premalignas tienen $100 \%$ de especificidad y $50 \%$ de sensibilidad, las lesiones malignas presentaron $50 \%$ de sensibilidad y $100 \%$ de especificidad. Hubo una sensibilidad muy baja de la histeroscopia para diagnóstico de hiperplasias y pólipos.

Conclusión: La ecografía tiene excelente eficacia para el diagnóstico de miomas submucosos. La histeroscopia es muy específica pero poco sensible para el diagnóstico de hiperplasias, pólipos, y lesiones premalignas.
\end{abstract}

Palabras clave: Pólipo endometrial, Mioma submucoso, Patología endometrial, Histeroscopia, Ecografía.

\section{SUMMARY}

Objective: To determine the diagnostic efficacy of ultrasound and hysteroscopy in patients with endometrial pathology who attended the gynecological service of the Hospital Universitario "Dr. Pedro Emilio Carrillo" January 2017- July 2017.

Method: A correlational, quasi-experimental and prospective study was conducted, including 33 patients with endometrial pathology who had transvaginal ultrasound, hysteroscopy and sampling for histological study.

Results: The average age of patients was $50 \pm 0.23$ years (33 - 72 years), the average age of menarquia was 11.84 years; obstetric history had the following means: gestations: $2.27 \pm 2,15$; $1.72 \pm 2.12$; abortions: $0.09 \pm 0.38$; $C$-section: $0.45 \pm 0.97$. The menstrual cycle was irregular in $54.5 \%$; $81.8 \%$ reported dysmenorrhea and $63.6 \%$ used oral contraceptives. Most of the patients reported having pelvic pain and a family history as a risk factor. The most frequent ultrasound diagnosis was endometrial thickening. The most common hysteroscopic diagnosis was submucosous myoma (36.4\%). Transvaginal ultrasound for the diagnosis of submucosal fibroids has $100 \%$ specificity and sensitivity; hysteroscopy has $96 \%$ specificity for endometrial polyp, premalignant lesions have $100 \%$ specificity and $50 \%$ sensitivity, malignancies had 50\% sensitivity and $100 \%$ specificity. There was a very low sensitivity of hysteroscopy for diagnosis of hyperplasms and polyps.

Conclusion: Ultrasound has excellent efficacy for diagnosing submucosous fibroids. Hysteroscopy is very specific but has a very low sensitivity for the diagnosis of hyperplasms, polyps, and premalignant lesions.

Keywords: Endometrial polyp, Submucosal myoma, Endometrial pathology, Hysteroscopy, Ultrasound.

\footnotetext{
'Departamento de Obstetricia y Ginecología del Hospital Universitario "Dr. Pedro Emilio Carrillo". ${ }^{2}$ Departamento de Obstetricia y Ginecología del Hospital Universitario "Dr. Pedro Emilio Carrillo". Profesora de la Universidad de los Andes (ULA). ${ }^{3}$ Departamento de Obstetricia y Ginecología del Hospital Universitario "Dr. Pedro Emilio Carrillo". ${ }^{4}$ Coordinadora Académica y profesora de Farmacología de la Escuela de Medicina ULA.

* Trabajo Especial de Grado presentado por María Blanco para optar al título de Especialista en Obstetricia y Ginecología. Universidad de los Andes.
}

\section{INTRODUCCIÓN}

Las patologías endometriales son una de las causas más frecuentes de consulta ginecológica, el estudio de estas patologías va dirigido principalmente a descartar 


\section{EFICACIA DIAGNÓSTICA DE LA ECOGRAFÍA Y LA HISTEROSCOPIA \\ EN PACIENTES CON PATOLOGÍA ENDOMETRIAL}

o en su defecto confirmar la patología del endometrio, con especial énfasis en el reconocimiento precoz del cáncer (1).

Entre las patologías orgánicas frecuentes están el pólipo endometrial, el mioma submucoso, la hiperplasia endometrial y el cáncer de endometrio. En mujeres posmenopáusicas que presentan sangrado uterino anormal, el $10 \%$ tendrán cáncer de endometrio; en las últimas décadas el cáncer de endometrio ha aumentado su incidencia, siendo actualmente el primer cáncer de origen ginecológico en los países desarrollados (2).

En Venezuela, para el año 2012, de acuerdo con la estimación estadística del sistema de registro central de cáncer, ocurrieron 42046 casos, en donde los tumores ginecológicos representaron el $1,97 \%$ de todas las causas de muerte, afectando con mayor frecuencia al grupo etario entre 55 y 64 años de edad, además, se reportaron 223 muertes por tumores del cuerpo uterino. El adenocarcinoma de endometrio representa la decimosegunda causa de muerte por cáncer ginecológico, precedido por el cáncer de la glándula mamaria, cuello uterino, pulmón, ovario y cáncer de colon (3).

Las mujeres que presentan sintomatología sospechosa de patología endometrial, son estudiadas con diferentes métodos complementarios que estiman el diagnóstico en un porcentaje variable, los más usados actualmente son la ecografía transvaginal y el legrado uterino fraccionado. En aquellas mujeres en las cuales el diagnóstico es todavía incierto, la histeroscopia con biopsia dirigida es el gold standard ya que detecta lesiones endometriales focales (pólipos, miomas submucosos) con mayor sensibilidad y especificidad, permitiendo obtener tejido para analizar (4).

Catalán y cols. (5), en 2007, reportaron una sensibilidad del ultrasonido para detectar cualquier patología endometrial del $92 \%$ y $96 \%$ para cáncer de endometrio. La especificidad es baja y lo es, aún más, en pacientes con terapia de reemplazo hormonal. Revisiones recientes confirman el uso de la ecografía transvaginal como instrumento útil para excluir patología endometrial cuando el límite de grosor que se usa es $5 \mathrm{~mm}$. Alfonso-Pérez y cols. (6), en 2009, señalaron que el sangrado uterino irregular es un gran problema durante el periodo perimenopáusico y la causa más común de sangrado irregular durante este periodo es la disminución de la función ovárica y la anovulación. Sin embargo, además de las disfunciones hormonales, las neoplasias endometriales son una causa importante de sangrado irregular y es necesario detectarlas, pero, a diferencia del cáncer de cuello uterino, los carcinomas endometriales son poco comunes en las mujeres menores de 40-45 años.

En condiciones normales, el endometrio presenta una medida específica según la fase del ciclo en el que se encuentre, variando en su fase proliferativa de 4 a 8 milímetros y en la fase secretora de 8 a 16 milímetros $(7,8)$. El ciclo endometrial consta de 3 fases $(7)$ :

1. Fase proliferativa (días 5-13 del ciclo), es la fase estrogénica del ciclo sexual femenino y ocurre antes de la ovulación. Al comienzo de cada ciclo mensual, parte del endometrio se descama por la menstruación, permaneciendo la capa basal. Bajo la influencia de los estrógenos que segregan los ovarios, las células del estroma y las células epiteliales proliferan reepitelizándose la superficie endometrial en 4 a 7 días desde el inicio de la menstruación.

2. Fase secretora o progestacional (días 14-28 del ciclo) después de la ovulación, son secretadas grandes cantidades de progesterona y de estrógenos por el cuerpo lúteo. En el momento culminante de la fase secretora (aproximadamente una semana después de la ovulación), el endometrio tiene un espesor de 5 a $6 \mathrm{~mm}$.

3. Menstruación (días 1-4 del ciclo), debida a la 
reducción repentina de estrógenos y progesterona, al final del ciclo ovárico, tras la involución del cuerpo lúteo, se produce la disminución de la estimulación de las células endometriales y luego la involución del endometrio.

Entre las patologías que producen afectación endometrial, se encuentra el mioma uterino, que es la neoplasia benigna más frecuente del útero y está constituido por fibra muscular lisa con un estroma conjuntivo en cantidad variable (8), aunque es de origen miometrial, cuando su ubicación es submucosa puede producir sangrado anormal. La causa exacta de la aparición de los miomas es, como en la mayoría de las neoplasias, desconocida. Sin embargo, existen cambios genéticos adquiridos por las células del miometrio que posteriormente se ven influenciadas por promotores (hormonas) y efectores (factores de crecimiento) $(8,9)$.

Los pólipos endometriales son formaciones sésiles o pediculadas que se proyectan desde la superficie endometrial, están constituidos por una proliferación de endometrio, glándulas y estroma, organizado sobre una raíz vascular. Esta proliferación localizada del endometrio raramente posee focos de crecimiento neoplásico, diagnosticándose cáncer en $5 \%$ de los pólipos resecados y analizados $(9,10)$.

La hemorragia uterina anormal(HUA) es definida como cualquier sangrado que difiera del patrón menstrual normal. Es la segunda causa de consulta ginecológica, después de las infecciones cérvicovaginales; su prevalencia es del $30 \%$, a tal punto que ocasiona $20 \%$ de las consultas en ginecología y $25 \%$ de todas las cirugías ginecológicas ( $65 \%$ de las histerectomías y $100 \%$ de la ablación endometrial). La frecuencia de las distintas causas de la HUA, varía según la edad de la paciente, el $50 \%$ se presentan en mujeres mayores de 45 años, un $30 \%$ entre los 20 a 44 años, $19 \%$ en adolescentes y $1 \%$ en premenárquicas $(11,12)$.
La hiperplasia endometrial (HE) se define como una proliferación de glándulas, de forma y tamaño irregular, con un incremento en la relación glándula/ estroma si se compara con el endometrio proliferativo normal. Este proceso suele ser difuso, pero no siempre afecta a toda la mucosa, se relaciona con el estímulo prolongado de estrógenos no compensado con gestágenos, siendo una lesión estrógeno-dependiente. El signo principal de la $\mathrm{HE}$ es la hemorragia uterina anormal (HUA), aunque puede cursar de manera asintomática (13).

La hiperplasia endometrial está clasificada como simple o compleja, también está clasificada si tiene células anormales presentes o ausentes y si presentan cambios anormales se denomina atípica. Se combinan los términos para describir el tipo exacto de hiperplasia: hiperplasia simple, compleja, simple con atipias y compleja con atipias (13). El riesgo de que la hiperplasia progrese a cáncer es bajo en el caso de hiperplasia simple y aumenta en el caso de hiperplasia compleja atípica. Se ha determinado que $42 \%$ de las mujeres con hiperplasia atípica pueden tener adenocarcinoma al momento de la cirugía (14).

La endometritis se refiere a la infección del endometrio o decidua, con potencial extensión al miometrio. El hecho de que la enfermedad se exprese o no clínicamente depende de la presencia de factores predisponentes, duración de la contaminación uterina, tipo y número de bacterias inoculadas. La necrosis decidual y otros productos como los loquios, promueven la proliferación de bacterias anaerobias (15).

Para el diagnóstico de las patologías endometriales, la anamnesis y la exploración física pueden resultar útiles para establecer una sospecha diagnóstica y las pruebas de imagen, especialmente la ecografía, son fundamentales para llegar al diagnóstico definitivo (9). 


\section{EFICACIA DIAGNÓSTICA DE LA ECOGRAFÍA Y LA HISTEROSCOPIA \\ EN PACIENTES CON PATOLOGÍA ENDOMETRIAL}

La ecografía transvaginal es la técnica más eficaz y de menor costo, es un procedimiento que utiliza ondas de sonido de alta frecuencia para producir una imagen de las estructuras pélvicas. Este es el método de diagnóstico por imágenes más comúnmente utilizado para observar los órganos pélvicos y no implica el uso de radiación; a menudo, se realizan ecografías tanto de la parte superior del abdomen y la pelvis como del interior de la vagina $(9,16)$. También pueden usarse la sonohisterografía (SHG), la resonancia magnética, la biopsia endometrial de consultorio, para examinar una muestra del endometrio que permite descartar anomalías cancerosas (17), y la histeroscopia, que es la técnica endoscópica que constituye el método diagnóstico y terapéutico de la cavidad uterina. Mediante la histeroscopia se puede diferenciar entre miomas, pólipos e hiperplasias, además, puede permitir identificar áreas específicas del endometrio en las cuales se puede realizar una biopsia $(18,19)$.

\section{MÉTODOS}

Fue un estudio correlacional, cuasi-experimental y prospectivo, conformado por todas las pacientes que cursaron con patología endometrial y que acudieron a la consulta de Ginecología del Hospital Universitario "Dr. Pedro Emilio Carrillo" durante el periodo enero a julio de 2017. Se incluyeron aquellas mujeres en edad fértil o posmenopáusicas, con 35 años de edad o más, diagnosticadas con patologías endometriales, quienes aceptaron participar en la investigación y firmaron un consentimiento informado. Se excluyeron mujeres con diagnóstico previo de cáncer endometrial, embarazadas y puérperas, aquellas a quienes se les había practicado legrado uterino reciente por aborto y mujeres que padecían enfermedad inflamatoria pélvica. Se contó con la autorización del Comité de Ética y Jefe de Departamento. A todas se les practicó la ecografía transvaginal con un equipo General Electric, modelo LOGIQ 3, imagen bidimensional con transductor 3,5 o 6,5 MHz. Una vez realizado el ultrasonido, se realizó el estudio histeroscópico con un equipo STORZ, (Karl
Storz - endoskope, tele pack X 200450 20), previa vaginoscopia y sin espéculo. Se envió muestra para análisis anatomopatológico.

Los datos se procesaron utilizando el programa informático de Microsoft Office Excel. Se calculó con medida de dispersión y tendencia central, mediante la prueba de Chi cuadrado de Pearson, Test exacto de Fisher, y prueba de $T$ de Student para muestras relacionadas. Se obtuvo Riesgo Relativo e intervalos de confianza al 95\%. Se consideró estadísticamente significativo todo valor de $\mathrm{p} \leq 0,05$.

\section{RESULTADOS}

El total de pacientes incluidas fue de 33. En la tabla 1, se evidencia que la edad media de las pacientes es de 50,75 $\pm 10,23$ años, con un rango de 33 a 72 años; 78,8 \% provienen de la zona urbana y $21,2 \%$ restante son de la zona rural; $33,3 \%$ de la población realizó estudios universitarios, mientras que $36,4 \%$ obtuvo educación diversificada y tan solo 30,3 \% tuvo educación básica,

Tabla 1. Características sociodemográficas

\begin{tabular}{lcc}
\hline Parámetro & Rango & $\mathrm{X} \pm \mathrm{DE}$ \\
\hline Edad (años) & $33-72$ & $50,75 \pm 10,23$ \\
Estado civil & Frecuencia & $(\%)$ \\
Soltera & 8 & 24,2 \\
Casada & 20 & 60,2 \\
Concubinato & 5 & 15,2 \\
Grado de instrucción & Frecuencia & $(\%)$ \\
Básica & 10 & 30,3 \\
Diversificado & 12 & 36,4 \\
Educación superior & 11 & 33,3 \\
Procedencia & Frecuencia & $(\%)$ \\
Urbana & 26 & 78,8 \\
Rural & 7 & 21,2 \\
\hline
\end{tabular}


además hubo predominio del estado civil casada $60,2 \%$.

Los antecedentes ginecobstétricos se presentan en la tabla 2. Se encontró que la edad promedio de la menarquia fue de 11,84 $\pm 2,22$ años; así mismo el promedio en gestaciones fue 2,27 $\pm 2,15$ embarazos. Las pacientes presentaron un ciclo menstrual regular en un $45,5 \%$ e irregular en un $54,5 \%$, y en cuanto a las características de la menstruación el $81,8 \%$ presentaron dismenorrea; $63,6 \%$ refirió uso de anticonceptivos orales.

La tabla 3 recoge las características clínicas de las pacientes. Hubo sangrado uterino anormal en el $54,5 \%$, sangrado posmenopáusico el $42,5 \%$, dolor

Tabla 2. Antecedentes ginecoobstétricos

\begin{tabular}{lcc}
\hline Parámetro & Rango & $\mathrm{X} \pm \mathrm{DE}$ \\
\hline Menarquia (edad/años) & $9-17$ & $11,84 \pm 2,22$ \\
Gestas & $0-8$ & $2,27 \pm 2,15$ \\
Para & $0-7$ & $1,72 \pm 2,12$ \\
Aborto & $0-2$ & $0,09 \pm 0,38$ \\
Cesárea & $0-3$ & $0,45 \pm 0,97$ \\
Duración de menstruación (días) & $3-5$ & $4,48 \pm 1,48$ \\
Ciclo menstrual & Frecuencia & Porcentaje \\
Regular & 15 & 45,5 \\
Irregular & 18 & 54,5 \\
FUR & Frecuencia & Porcentaje \\
Precisa & 13 & 39,4 \\
Imprecisa & 20 & 60,6 \\
Característica de menstruación & Frecuencia & Porcentaje \\
Eumenorrea & 6 & 18,2 \\
Dismenorrea & 27 & 81,8 \\
Uso de ACO & Frecuencia & Porcentaje \\
Si & 21 & 63,6 \\
No & 12 & 36,4 \\
\hline
\end{tabular}

FUR: fecha de última regla; ACO: anticonceptivos orales.
Tabla 3. Características clínicas

\begin{tabular}{lcc}
\hline Características clínicas & Frecuencia & Porcentaje (\%) \\
\hline Dolor pélvico & 27 & 81,8 \\
Sangrado uterino anormal & 18 & 54,5 \\
Sangrado posmenopáusico & 14 & 42,4 \\
Anemia & 10 & 30,3 \\
\hline
\end{tabular}

pélvico en el $81,8 \%$, y solo el $30,3 \%$ presentó anemia durante el desarrollo de su patología.

En la tabla 4 se observa que el factor de riesgo que se presentó con mayor frecuencia fue el antecedente familiar de patología endometrial $(69,7 \%)$, seguido de la obesidad $(42,4 \%)$; otros factores que se presentaron con frecuencia fueron el consumo de tabaco (36,4\%), la nuliparidad (27,3\%), menarquia temprana $(18,2 \%)$, menarquia tardía $(15,2 \%)$ y solo un $12,1 \%$ presentó como factor de riesgo la edad avanzada.

En la tabla 5 están los hallazgos al ecosonograma: el $45,5 \%$ fue diagnosticada con engrosamiento endometrial, seguido del mioma submucoso con $36,4 \%$ y $18,2 \%$ pólipo endometrial. Los diagnósticos histeroscópicos se presentan en la tabla 6 , donde

Tabla 4. Factores de riesgo

\begin{tabular}{lcc}
\hline Factor de riesgo & Frecuencia & Porcentaje (\%) \\
\hline Antecedente familiar & 23 & 69,7 \\
Obesidad & 14 & 42,4 \\
Tabaco & 12 & 36,4 \\
Nuliparidad & 9 & 27,3 \\
Menarquia temprana & 6 & 18,2 \\
Menarquia tardía & 5 & 15,2 \\
Edad avanzada & 4 & 12,1 \\
\hline
\end{tabular}




\section{EFICACIA DIAGNÓSTICA DE LA ECOGRAFÍA Y LA HISTEROSCOPIA \\ EN PACIENTES CON PATOLOGÍA ENDOMETRIAL}

Tabla 5. Hallazgos de la ecografía transvaginal

\begin{tabular}{lcc}
\hline Hallazgo ecográfico & Frecuencia & Porcentaje (\%) \\
\hline $\begin{array}{l}\text { Engrosamiento } \\
\text { endometrial }\end{array}$ & 15 & 45,5 \\
Mioma submucoso & 12 & 36,4 \\
Pólipo endometrial & 6 & 18,2 \\
\hline
\end{tabular}

Tabla 6. Hallazgos histeroscópicos

\begin{tabular}{lcc}
\hline Hallazgo histeroscópico & Frecuencia & Porcentaje (\%) \\
\hline Mioma submucoso & 12 & 36,4 \\
Pólipo endometrial & 10 & 30,3 \\
Engrosamiento & 9 & 27,3 \\
endometrial & 1 & 3,0 \\
Lesión premaligna & 1 & 3,0 \\
Lesión maligna & & \\
\hline
\end{tabular}

Tabla 7. Hallazgos histopatológicos

\begin{tabular}{lcc}
\hline Hallazgo histopatológico & Frecuencia & Porcentaje (\%) \\
\hline Mioma submucoso & 12 & 36,4 \\
$\begin{array}{l}\text { Pólipo endometrial } \\
\text { Hiperplasia simple sin } \\
\text { atipias }\end{array}$ & 10 & 30,3 \\
$\begin{array}{l}\text { Hiperplasia compleja sin } \\
\text { atipias }\end{array}$ & 3 & 15,2 \\
$\begin{array}{l}\text { Adenocarcinoma } \\
\text { Hiperplasia simple con } \\
\text { atipias }\end{array}$ & 2 & 9,1 \\
\hline
\end{tabular}

Tabla 8. Concordancia los hallazgos ecográficos, histeroscópicos e histológicos

\begin{tabular}{lcc}
\hline Hallazgo & Frecuencia & Porcentaje \\
\hline $\begin{array}{l}\text { Ecografía + histeroscopia } \\
+ \text { histopatología }\end{array}$ & 18 & 54,5 \\
$\begin{array}{l}\text { Ecografía }+ \\
\text { histopatología }\end{array}$ & 9 & 27,3 \\
$\begin{array}{l}\text { Histeroscopia }+ \\
\text { histopatología }\end{array}$ & 6 & 18,2 \\
\hline
\end{tabular}

se puede ver que $36,4 \%$ presentaron mioma submucoso, 30,3\% pólipos endometriales, $27,3 \%$, engrosamiento endometrial, $3 \%$ presentó lesión premaligna y otro $3 \%$ sospecha de cáncer de endometrio.

La tabla 7 muestra los hallazgos histopatológicos, $36,4 \%$ presentó mioma submucoso, 30,3\% pólipo endometrial, 15,2 \% hiperplasia endometrial simple sin atipias, 9,1\% hiperplasia endometrial compleja sin atipias, $3 \%$ hiperplasia endometrial simple con atipia y $6,1 \%$ de las pacientes fueron diagnosticadas con adenocarcinoma.

La tabla 8, muestra la concordancia entre los hallazgos ecográficos, histeroscópicos con la histología. La relación diagnóstica entre la ecografía, la histeroscopia y el resultado histopatológico fue de 54,5\%; entre la ecografía y la histopatología, en $27,3 \%$ y entre la histeroscopia y el resultado histopatológico en 18,2\%.

En la tabla 9, se presentan todos los índices de eficacia de los dos métodos. Destaca que para la ecografía, el diagnóstico de mioma submucoso tiene $100 \%$ de especificidad y sensibilidad, mientras que para el diagnóstico de pólipo endometrial se evidenció un $60 \%$ de sensibilidad y $100 \%$ de especificidad y para el diagnóstico de engrosamiento endometrial la sensibilidad fue de $100 \%$ y la especificidad del $75 \%$. Para la histeroscopia, la sensibilidad fue de 0 para los diagnósticos de hiperplasia compleja sin atipias, hiperplasia simple con atipias y pólipo endometrial. La especificidad fue de $96 \%$ para los tres diagnósticos. Para lesión premaligna, la sensibilidad fue de $50 \%$ y la especificidad de $100 \%$ y para adenocarcinoma, la sensibilidad fue de $50 \%$ y la especificidad de $1 \%$. 
M BLANCO ET AL.

Tabla 9. Eficacia de ecografía, histeroscopia en pacientes con patología endometrial

\begin{tabular}{lcccccc}
\hline & $\mathrm{S}$ & $\mathrm{E}$ & $\mathrm{VPP}$ & $\mathrm{VPN}$ & $\mathrm{V}+$ & $\mathrm{V}-$ \\
\hline Ecografía & & & & & & \\
$\quad$ Mioma submucoso & 100 & 100 & 1 & 1 & 1 & - \\
$\quad$ Pólipo endometrial & 60 & 100 & 1 & 0,85 & 0,6 & 0,6 \\
$\quad$ Engrosamiento endometrial & 100 & 75 & 0,6 & 1 & 0,4 & 0,75 \\
Histeroscopia & & & & & & \\
$\quad$ Hiperplasia compleja sin atipias & 0 & 96 & 0 & 0,96 & 0 & 0 \\
Pólipo endometrial & 0 & 96 & 0 & 0,84 & 0 & 0 \\
Lesión premaligna & 50 & 100 & 0 & 0,90 & 0,5 & 0 \\
Hiperplasia simple con atipias & 0 & 96 & 0 & 0,84 & 0 & 0,4 \\
Adenocarcinoma & 50 & 1 & 1 & 0,96 & 0,5 & 0 \\
\hline
\end{tabular}

S: sensibilidad; E: especificidad; VPP: valor de predicción positivo; VPN: valor de predicción negativo; $\mathrm{V}+$ : coeficiente de variabilidad positivo; $\mathrm{V}$-: coeficiente de variabilidad negativo.

\section{DISCUSIÓN}

En esta investigación se evidenció que la edad media que con mayor frecuencia presenta patología endometrial fue de 50,75 \pm 10,23 años; esto se relaciona con los resultados de Catalán y cols. (5) quienes encontraron una edad promedio de 54 años, con un rango entre 26 y 85 años, 63 pacientes (64 \%) eran posmenopáusicas y 35 pacientes $(36 \%)$ premenopáusicas.

La característica clínica que con mayor frecuencia presentaron las pacientes en este estudio fue sangrado uterino anormal con un 54,5\%, esto se relaciona con lo expuesto por Iglesias y cols. (9), quienes describieron que el sangrado en pacientes con miomatosis uterina es el signo más frecuente, produciendo un aumento en la cantidad y duración de la menstruación, esto último es más frecuente en el caso de miomas submucosos y en pacientes con otras entidades como hiperplasias. Si bien el mioma submucoso no es una patología propia del endometrio, por ser una lesión miometrial, la manifestación clínica más frecuente es el sangrado uterino anormal, sobre todo cuando es de ubicación submucosa. Manrique (13) determinó que 8 de cada 1000 mujeres posmenopáusicas que cursan con hiperplasia endometrial son asintomáticas y en un
$15 \%$ presentan sintomatología; así mismo describió que las patologías endometriales tipo hiperplasia endometrial sin atipias, se presentan con mayor frecuencia en mujeres posmenopáusicas, mientras que la hiperplasia endometrial con atipias (HEA) es más frecuente en mujeres a partir de los 60 años. En esta investigación, el 42,5\% de las pacientes presentaron sangrado posmenopáusico. Se observó que $81,8 \%$ de las pacientes presentaron dolor pélvico, frecuencia más elevada que la descrita por Iglesias y cols. (9) quienes describieron que el $30 \%$ de las mujeres con miomas u otra patología endometrial pueden presentar dolor pélvico crónico o persistente y esto puede deberse a cambios degenerativos con necrosis. Por su parte, De la Fuente y cols. (10) demostraron que las pacientes con pólipos endometriales presentaban dolor pélvico por producir dilatación cervical y la hemorragia uterina era más intensa. Una tercera parte de las pacientes presentó anemia durante el desarrollo de su patología.

El factor de riesgo que se presentó con mayor frecuencia fue el antecedente familiar, con un $69,7 \%$, concordando con Iglesias y cols. (9) quienes describieron que las mujeres con familiares de primer grado afectadas con patologías endometriales tienen mayor riesgo de desarrollar las mismas, así mismo 


\section{EFICACIA DIAGNÓSTICA DE LA ECOGRAFÍA Y LA HISTEROSCOPIA \\ EN PACIENTES CON PATOLOGÍA ENDOMETRIAL}

se observó que $42,4 \%$ de las pacientes presentaban obesidad, coincidiendo con lo descrito por Manrique (13), quien reportó que las pacientes obesas eran más propensas a desarrollar hiperplasia endometrial. En relación a los miomas, Iglesias y cols. (9) describieron que no existen estudios que se relacionen con la aparición de miomas en pacientes obesas.

Otro factor de riesgo presente fue el consumo de tabaco con $36,4 \%$, con respecto a este punto, Iglesias y cols. (9) describieron que en las mujeres fumadoras puede estar reducida la incidencia de miomas y esto puede deberse a una disminución de la biodisponibilidad de los estrógenos, secundaria a una disminución de la actividad de la aromatasa. También se observó que las pacientes nulíparas desarrollaron patologías endometriales en un 27,3\%, coincidiendo con Manrique (13), quien describió que las nulíparas son más propensas a desarrollar patologías endometriales, con mayor énfasis en el desarrollo de hiperplasia endometrial. Hubo un 18,2 \% de menarquia temprana; Iglesias y cols. (9) señalaron que las mujeres que tienen una menarquia temprana tienen un riesgo de padecer miomatosis uterina entre un $25 \%-50 \%$, mayor que aquellas que tienen su menarquia después de los 13 años de edad. Otro factor de riesgo que estuvo presente en la investigación fue la edad avanzada con un $12,1 \%$, Manrique (13), describió que las pacientes con edad avanzada tenían mayor predisposición a desarrollar hiperplasia endometrial, mientras que Iglesias y cols. (9), describieron que las pacientes que a los 40 años de edad no desarrollen miomatosis uterina, difícilmente lo hagan en edades más avanzadas.

Entre las 33 pacientes que participaron en esta investigación se logró identificar que 36,4 \% fueron diagnosticadas con mioma submucoso. Iglesias y cols. (9) describieron que en un $50 \%$ de mujeres con miomatosis uterina, el diagnóstico será un hallazgo casual en la ecografía realizada por otra causa. De ahí la importancia de la adecuada evaluación de las pacientes con sangrados anormales. Se logró identificar que $45,5 \%$ fueron diagnosticadas con engrosamiento endometrial a través de la ecografía transvaginal. Crivelli y cols. (4) demostraron que la ecografía es uno de los métodos más utilizados para evaluar el endometrio, su espesor, homogeneidad y lesiones focales. Es una técnica económica, bien tolerada por las pacientes, sin complicaciones y reproducible. El $18,4 \%$ fueron diagnosticadas con pólipos endometriales. Franco (12), en 2012, reportó que el uso de la ecografía en ginecología ha aumentado de forma considerable el diagnóstico de sospecha de pólipo endometrial, incluso en pacientes asintomáticas. Ello ha llevado al dilema de qué hacer con el gran número de diagnósticos de sospecha ecográfica de pólipo endometrial, que se encuentran en la práctica clínica diaria.

Todas las pacientes fueron sometidas a un estudio histeroscópico. Los resultados se relacionan con lo expuesto por Franco (12) quien describió que hay clara evidencia de que la histeroscopia es la técnica de elección para confirmar o no la sospecha diagnóstica, así como confirmar la naturaleza y localización de la lesión. Crivelli y cols. (4) encontraron una concordancia aceptable entre la ecografía y la histeroscopía en el caso de las lesiones focales.

Una vez realizada la histeroscopia, se tomaron muestras de las lesiones encontradas; los diagnósticos fueron, en orden de frecuencia, miomas submucosos, pólipos endometriales, hiperplasia endometrial simple sin atipias, hiperplasia endometrial compleja sin atipias, hiperplasia endometrial simple con atipia $\mathrm{y}$ adenocarcinoma.

Se pudo constatar que en $27,3 \%$ de los casos hubo relación diagnostica entre la ecografía y la histopatología $\mathrm{y}$ en $18,2 \%$ hubo relación entre histeroscopia e histopatología. Asimismo, en el 54,5 \% de la población estudiada hubo relación diagnostica entre los tres estudios. Esto demuestra la importancia del rol confirmatorio del análisis histopatológico sobre las patologías benignas y/o malignas. 
Al evaluar los índices de eficacia destacan la elevada sensibilidad y especificidad de la ecografía para los tres diagnósticos considerados, sin embargo, llama la atención la sensibilidad de 0 para el diagnóstico histeroscópico de hiperplasias y pólipos. Crivelli y cols. (4) demostraron que el estudio de la patología endometrial puede realizarse por distintos métodos complementarios, teniendo la ecografía transvaginal una sensibilidad de $92 \%$ a $96 \%$ y una especificidad de $61 \%$ a $88 \%$. En contraste, la histeroscopia presenta una sensibilidad de $75 \%$ a $86 \%$ y una especificidad de $96 \%$ a $99 \%$ para la detección de patología endometrial focal (pólipos y miomas) y explora la cavidad endometrial en forma completa, permitiendo dirigir la toma de biopsia, lo que es útil en el diagnóstico de lesiones benignas y malignas.

\section{REFERENCIAS}

1. Socías M, Vargas R, Costoya A, Monje B, Haye M. Correlación de la histeroscopia y biopsia dirigida en el estudio del engrosamiento endometrial patológico por ultrasonido. Rev Chil Obstet Ginecol [Internet]. 2007 [consultado 17 enero de 2017]; 72(2):99-104. Disponible en: https://scielo.conicyt.cl/pdf/rchog/ v72n2/art06.pdf

2. Raby T, Sierra P, Tsunekawa H, Craig J, Carvajal J, Cuello M. Capacidad diagnóstica de la ecografía para detectar cáncer de endometrio en mujeres posmenopáusicas sintomáticas y asintomáticas: experiencia de la Universidad Católica de Chile. Rev Chil Obstet Ginecol [Internet]. 2014 [consultado 16 enero de 2017]; 79(3):166-172. Disponible en: https:// scielo.conicyt.cl/pdf/rchog/v79n3/art04.pdf

3. Capote L. Resumen de las estadísticas de cáncer en Venezuela. Sociedad Venezolana de Salud Pública. Red Defendamos la Epidemiología Nacional [Internet]. Caracas: Sociedad de Obstetricia y Ginecología de Venezuela; 2015 [consultado 20 enero de 2017]. Disponible en: http://www.sogvzla. org.ve/sogvzla20186/saciverfotonoticiaconvista. php?id $=0000000142$

4. Crivelli R, Bacigaluppi A, Bressan M, Avellino R, Uribe M, Sansó M. Correlación ecográfica, histeroscópica, y patológica en el estudio de patología endometrial. Rev Hosp Priv Com [Internet]. 2010 [consultado 20 febrero de 2017]; 13(1): 24-28. Disponible en: https:/hpc.org. ar/wp-content/uploads/759-24-CRIVELLI.pdf

5. Catalán A, Corvalán J, Pantoja V, García K, Godoy M. Pólipos endometriales: Manejo con histeroscopia quirúrgica y correlación histológica. Rev Chil Obstet Ginecol [Internet]. 2007 [consultado 17 enero de 2017]; 72(2):116-119. Disponible en: https://scielo.conicyt.cl/scielo.php? script $=$ sci_ arttext\&pid=S0717-75262007000200009

6. Alfonso-Pérez A, Navarro Y, Schloeter J, Delgado $\mathrm{O}$, Torres D, Santos J, et al. Ultrasonido transvaginal en premenopáusicas para el estudio de patología endometrial. MedULA [Internet]. 2009 [consultado 17 enero de 2017]. 18(2):129-133. Disponible en: http:// www.saber.ula.ve/bitstream/handle/123456789/30692/ articulo10.pdf? sequence $=2 \&$ is Allowed $=\mathrm{y}$

7. López M. Regulación neurológica y hormonal de la función reproductora. Fisiología de la pubertad y del climaterio [Internet]. Albacete: Complejo Hospitalario Universitario Albacete; 2012 [consultado 17 enero de 2017]. Disponible en: https://www.chospab. es/area_medica/obstetriciaginecologia/docencia/ seminarios/2012-2013/sesion20120620.pdf

8. Vidal J. Patología tumoral del cuerpo uterino. En: Usandizaga y De la Fuente editores. Ginecología. 4ta Edición. Cd México: Marbán; 2011. p 1103 - 1119.

9. Iglesias E, Espantaleón M. Mioma Uterino. En: Cabero L editor. Tratado de Ginecología y Obstetricia. Tomo I. $2^{\mathrm{a}}$ ed. Madrid: Médica Panamericana; 2012. p 719-729.

10. De la Fuente P, Garzón A. Patología endometrial benigna. En: Usandizaga y De la Fuente editores. Ginecología, 4ta Edición, Cd México: Marbán; 2011. p $1120-24$.

11. Elliott J, Connor ME, Lashen $\mathrm{H}$. The value of outpatient hysteroscopy in diagnosing endometrial pathology in postmenopausal women with and without hormone replacement therapy. Acta Obstet Gynecol Scand. 2003; 82(12):1112-1119. doi: 10.1046/j.16000412.2003.00261.x. PMID: 14616256.

12. Franco R. Hemorragia uterina anormal orgánica. Rev Nac (Itauguá) [Internet]. 2012 [consultado 26 julio de 2017]; 4(2):15-22. Disponible en: http://scielo.iics.una.py/scielo.php?script=sci arttext\&pid=S2072-81742012000200003

13. Manrique M. Hiperplasia endometrial tipos, diagnóstico y tratamiento [Internet]. Granada: Hospital Universitario Virgen de Las Nieves; 2009 [consultado 


\section{EFICACIA DIAGNÓSTICA DE LA ECOGRAFÍA Y LA HISTEROSCOPIA \\ EN PACIENTES CON PATOLOGÍA ENDOMETRIAL}

15 enero de 2017]. Disponible en: https://silo.tips/ download/hiperplasia-endometrial-tipos-diagnostico$\mathrm{y}$-tratamiento\#

14. Suh-Burgmann E, Hung YY, Armstrong MA. Complex atypicalendometrialhyperplasia:theriskofunrecognized adenocarcinoma and value of preoperative dilation and curettage. Obstet Gynecol. 2009; 114(3):523-529. doi: 10.1097/AOG.0b013e3181b190d5. PMID: 19701030.

15. León H, Valderrama A. Endometritis puerperal: diagnóstico, manejo y prevención [Internet]. Ciudad: Ginecol Hospital El Tunal; 2007 [consultado 17 enero de 2017]. Disponible en: https://nanopdf.com/ download/9-1-prevencion-de-infecciones_pdf

16. American Society for Reproductive Medicine (ASRM) [Internet]. Washington, DC: Sangrado Uterino Anormal; 2013 [consultado 16 enero de 2017]. Disponible en: https://www.asrm.org/topics/topics-index/abnormaluterine-bleeding/

17. Rodríguez C, Álvaro M, Rojo J, Moreno J, Castellanos P. Historia de la histeroscopia. En: Huertas M, Rojo $\mathrm{J}$, editores. Manual de histeroscopia diagnóstica y quirúrgica. Barcelona: Editorial Panamericana; 2008. p 17-25.
18. Cortés S, Álvarez V, Kishimoto C, Lineros E, Carballal J, Ojeda F. Correlación entre la histeroscopia diagnóstica de la patología endocavitaria [Internet]. Cataluña: Fundación Hospital Asil de Granollers; 2007 [consultado 15 de enero de 2017]. Disponible en: http:// e-archivos.org/e-AGO200803/correHSC_ECO.pdf

19. Gezer A, Şaar A, Demirkiran F, Benian A, Şimşek Y, Albayrak M, Kösebay D. The efficacy of hysteroscopy for endometrial pathology: the experience of a university clinic on diagnostic accuracy and the comparison with the other methods. Gynecol Surg. 2004; 1:227-230. https://doi.org/10.1007/s10397-004-0050-z

Recibido 1 de mayo de 2020 Aprobado 10 de septiembre de 2020 